\title{
Initial Results in Developing an Engineering Reasoning Assessment for Gen- eral Education
}

\section{Dr. John Krupczak Jr, National Science Foundation}

Professor of Engineering, Hope College, Holland, Michigan. Former Chair of the ASEE Technological Literacy Division. Former Chair of the ASEE Liberal Education Division. Senior Fellow CASEE, National Academy of Engineering, 2008-2010.

\section{Dr. Mani Mina, Iowa State University}

Mani Mina is with the department of Electrical and Computer Engineering at Iowa State University. He has been working on better understanding of students' learning and issues of technological and engineering philosophy and literacy. In particular how such literacy and competency are reflected in curricular and student activities. 


\section{Initial Results in Developing an Engineering Reasoning Assessment for General Education}

Despite the importance of technology to our well-being and the significance of engineering principles to economic prosperity, limited work has been done measuring the degree to which undergraduate students possess a broad understanding of the principles, products, and processes of technology. While assessments of learning gains within courses that form part of an engineering major have been developed under ABET EC 2000, the means of assessing the technological understanding of the majority of undergraduates who are not engineering students is yet to be systematically addressed. The work reported here describes the initial stages of an effort to develop a technological literacy assessment suitable for use with students who are not intending careers in STEM disciplines. As an initial starting point, the work focuses on abilities related to the identification and characterization of systems. Specific abilities include identifying a system and its boundaries, recognition of inputs and outputs, analysis of system structure, determination of subfunctions within the system, recognition of major components, associating specific physical processes with particular components, and discernment of the boundaries of the system. The ability to synthesize systems to achieve a particular function in given technological domain is also considered. This work begins an effort to create some assessment tool appropriate for use with the large number of students who are not majoring in one of the STEM disciplines. Preliminary from using these assessments with undergraduate non-STEM majors are described.

\section{Background}

Our modern economy and way of life depend on the use of technology. In Technically Speaking: Why All Americans Need to Know More about Technology, ${ }^{1}$ the National Academy of Engineering (NAE) has made an effort to publicize and clarify the importance of technology in our daily lives. Informed citizens should possess an understanding of technology, how it is developed, how it works, how it affects society, and how society determines the path of technological developments.

Engineering and technological literacy are defined as an ability to understand the broader technological world. The National Academy of Engineering has defined technology is any modification made to the natural world to meet a human need or want. Technology includes physical products, but also processes and knowledge needed to develop and apply these products. The facilities and expertise needed in the design, manufacture, operation, and repair of technological devices and systems are also considered as part of our technological infrastructure.

\section{Assessment of Engineering and Technological Literacy}

While the importance of engineering and technological literacy is well recognized, no widely used method is available to measure or characterize the broad understanding of the human-built environment by people who are not engineers or other technical professionals. Learning outcomes exist for those obtaining accredited engineering degrees due to the ABET accreditation 
criteria. No convenient means exists for assessing the understanding of our technological world by the majority of citizen who are not STEM professionals.

The NAE Committee on Assessing Technological Literacy carried out a study of available methods for measuring technological and engineering literacy. The results of this study were reported in Tech Tally ${ }^{2}$ in 2006. The committee concluded that few means existed to measure the broader understanding of technology among the general population and no appropriate, broadlybased assessment instrument yet exists. The NAE stated: "Thus far, no studies have addressed general engineering concepts, such as systems, boundaries, constraints, trade-offs, goal setting, estimation, and safety." It further added: "Not a single study investigates what the general public understands about these concepts, much less how they come to understand them.”

\section{Overview of Current Effort}

The work reported here seeks to develop some means of assessing the understanding of modern technological systems by people who have not received specific training about these systems as part of a job or career. A particular focus will be undergraduate students in US colleges and universities. The assessment methods developed are expected to be suitable for use by faculty teaching general education courses on engineering and technological topics.

\section{Outline of Approach for Assessment}

A challenge facing those intending to develop tests of engineering and technological literacy is the vast amount of potential information that might be appropriate to include in such an assessment. An inventory of what everyone should know about engineering and technology would be an expansive list. Even one of the sub-disciplines of engineering such as mechanical, electrical, or civil engineering could produce a near limitless array of facts to include. Compounding the problem is the time evolution of technology. One only needs to look back 5 to 10 years to realize that the technological systems considered mandatory for the tech savvy citizen of today will be rapidly out-of-date. For this reason basing an assessment on a list of specific technological devices was not considered to be the best approach.

The approach used seeks to develop an assessment that is general with regard to the types of technological systems addressed and not dependent on extensive specific prerequisite knowledge, for example the use of calculus or statistics.

Characteristics similar to a concept inventory were considered a potentially useful in this instance. The most well-known example of this assessment approach is the Force Concept Inventory as developed by Hestenes, Wells, Swackhammer, and others ${ }^{3}$. Key features of concept inventory tests are the use of a multiple-choice question format, and inclusion of a variety of distractor options to help reveal the nature and extent of misconceptions. Concept inventories avoid questions that can be answered using rote skills. The length of the Force Concept Inventory is typical for these types of assessments. The Force Concept Inventory is 30 questions. 
Certain features of concept inventories render this approach less than ideal for measuring a broad understanding of the engineered world. First, concept inventories generally address a very specific and well-defined knowledge domain. The Force Concept inventory is not an inventory of all physics concepts but rather a subset of Newtonian mechanics. This narrow scope of concept inventories was seen as a potential limitation. Additionally, concept inventories once developed, are static. The possibility of changing over time is not included.

Other possibly more suitable models were identified in the Law School Admissions Test $(\mathrm{LSAT})^{4}$, the Miller Analogies Test (MAT) ${ }^{5}$ and the ACT Science Reasoning Test ${ }^{6}$. These tests are intended to measure skills and abilities in a particular area but do not assume specific prior factual knowledge. The LSAT measures a variety of abilities considered relevant to the practice of law. These include the ability to read complex lengthy materials, the ability to understand the structure of relationships and to draw logical conclusions and an ability to evaluate arguments. No prior legal training is assumed and the factual information needed to engage in these higher level cognitive tasks is included in the question.

The ACT Science Reasoning Test follows a similar approach. This test presents the test taker with passages that contain representations of data, research summaries, and/or hypothesis that may be mutually inconsistent. Questions focus on the ability to engage in scientific inquiry and include evaluating concepts, assessing assumptions and the components of an experimental design; drawing conclusions from data; and generating models consistent with given information. A key aspect of the ACT Science Test is a focus on scientific inquiry and reasoning rather than recall of specific facts learned prior to the test. With an emphasis on science reasoning, the subject matter of the questions can range across the wide assortment of science fields including: biology, botany, zoology, chemistry, physics, geology, astronomy, and meteorology.

The ACT Science Reasoning Test was taken as a model for developing an Engineering Reasoning test. The general approach would be to identify some aspects of engineering thinking that span all engineering fields just as the ACT Science Test identifies scientific inquiry skills that are common to the biological and physical sciences. An advantage of this approach is the ability to draw material from a wide range of engineering products and the potential to continually update the content just as the ACT Science Test can include many possible science topics.

At this point in the work, the term "Engineering Reasoning" was adopted as opposed to the terms “engineering" or "technological literacy." Like science reasoning, engineering reasoning points to a specific set of skills and abilities used across a particular subject matter domain. Engineering reasoning reduces many of the misunderstandings and ambiguities that have accompanied "literacy” terminologies.

\section{Framework for Engineering Reasoning}

A framework was adopted upon which the engineering reasoning test could be based. An important goal is the framework must apply to all engineering disciplines and not be specific to any one specialty of engineering. In addition the framework should include the ideas and 
perspectives that guide how engineering experts think about, and interact with technology. This framework has been described in detail in an earlier work ${ }^{7}$. The main themes which serve as a framework for the engineering of technology are summarized as follows:

1. Technology created for a function accomplished through form. Technology is created to solve problems and satisfy needs. The function is the problem to be solved or a need to be satisfied. Function, or what a particular object can do, is determined by the form or the physical properties and characteristics of that object. The form of any object can provide a variety of different functions.

2. Technological systems transform materials, energy, and information.

Technological products are systems that transform inputs into outputs. Inputs and outputs take the form of flows of materials, energy, and information.

3. Function is provided by components combined into systems.

Overall system function is provided by components which combine to form systems. The term "subassembly" is sometimes used to describe intermediate stages of component groups. Some components are utilized for the purposes of controlling system behavior.

4. Components utilize physical phenomena.

Components accomplish transformations through utilization of physical phenomena.

Transformations and component behavior are frequently expressed in mathematical form. Predictive capabilities of mathematics facilitate component interconnection and achievement of system performance requirements.

5. System employ diverse interacting phenomena.

Most technological systems utilize a diverse range of phenomena. Components utilizing different phenomena must interact through exchanging the same type of material, energy, or information flow.

6. Component functions transfer across systems.

Components can be used to provide the same subfunction in systems with different overall function. Component variations offer diverse features around the same core function and principle.

7. Systems become components and systems are sociotechnical.

There is no absolute distinction between a component and a technological system. One technological system can become a component in another system. Technological system boundaries are arbitrary and depend upon the intent of a particular analysis. Technological systems are sociotechnical in nature encompassing social and cultural interactions.

8. System design creates component ensembles with emergent properties. Systems result in utility exceeding that of individual components in aggregate. Technological system design envisions function structures to achieve overall system function. Components solve subproblems or subfunctions within the system. Component parameters are adjusted in accordance with desired features, system performance, and requirements of the intracomponent 
interactions. The design process employs form and function representations of the system. System designs require compromise between competing objectives.

9. Technological system domains are groups of related systems.

Engineering design domains exist around collections of related systems. Systems within a domain typically provide the same overall function in diverse applications. Systems within a domain share common components and core underlying principles. Refrigeration systems is an example of a domain. Specific systems within the domain would include domestic and commercial refrigerators and freezers of various sizes and capacities, special purpose refrigerated systems like bottled drink vending machines, and refrigeration systems adapted for mobile use in transport and shipping applications.

10. Technological systems evolve.

Technological systems frequently evolve through substitution at the component level. Specific modes include merging of separate components into integral structures, optimization of component features, proliferation of added subfunctions, and substitution of component operating principles.

\section{Trial Test Development}

The initial test was not intended to comprehensively address the nature of technology and all aspects of how engineering experts think and reason about technology. Rather, the goal was to test the possibility of creating questions that do not rely on extensive specific prior knowledge and employ higher order thinking and reasoning skills. As in the case with the ACT Science Reasoning Test, by providing data and low-level details, the question can engage students at the Application and Analysis level of Bloom's Taxonomy of the Cognitive Domain rather than lower levels of Knowledge (Remembering) or Comprehension (Understanding Facts).

An initial test of seven questions was developed. An example question is included in Appendix A. In this section the intent of each question will be described.

Question 1: Design Using Components

The question addresses the issue that technological systems are created from components that provide a specific function and systems can have multiple modes of operation. The objective is to select an appropriate design for a hybrid car. Five major components are given: Battery, Fuel Tank, Internal Combustion Engine, Electric Motor, and Electric Generator. Operation is described for the case in which the battery is depleted and this hybrid uses the internal combustion engine and electric motor to recharge the battery. A correct systems-level diagram must be selected. Distractors include direct connection of the internal combustion engine and the wheels as well as inappropriate direction of energy transfer between some of the components.

Question 2: Components Utilize Physical Phenomena

This question uses the example of a refrigerator to test the association between the components of the system and the underlying physical principle or principles utilized in a particular component. The operation of the refrigerator as a system is described. The question asks the 
respondent to identify which component heat causes a change of state of the refrigerant from a liquid to a gas. The question take cares not to use the same words or terminology as was used in the system description.

Question 3: Form Follows Function

The idea that the form or physical characteristics of a component in an engineered system are selected to help accomplish the particular purpose of that component is addressed in this question. The refrigerator is again the system in question. The underlying physical principles employed in the condenser are explained. The question then asks the test taker to select which of a set of physical characteristics of the condenser are influenced by the underlying principles at work.

Question 4: Use of Mathematical Models in the Design Process

It is widely accepted that a defining characteristic of modern engineering is the use of mathematical models that describe the behavior of physical systems. This question involves the use of a mathematical formula to help select an appropriate component for a system. The component is an electric heater. The question provides the relation between power, voltage, and current $(\mathrm{P}=\mathrm{I} \mathrm{V})$. A specific heater must be chosen from an actual manufacturer's catalog.

This question is challenging because the catalog does not contain a heater that is an exact match to the parameters given in the question. The best fit is a heater with a slightly lower wattage than that calculated. The catalog excerpt also contains heaters of different voltage ratings beside that specified in the problem and other distracting information that must be ignored.

Question 5: Identification of System Inputs and Outputs.

This question addresses identification of the inputs and outputs of a technological system. A hairdryer is shown and the question asks for the most general description of the system inputs and outputs. The distracting choices include more common, but less general, inputs and outputs of this system.

Question 6: Technological Domains and Related Systems

Engineering experts can recognize when technological systems are similar based on the types of components and subsystems used rather than outward appearance or even end use. The purpose of this question is to test this reasoning ability. Five common kitchen appliances are given: coffeemaker, toaster, coffee grinder, microwave oven, and a blender. Diagrams are given for each device which show cut-away and exploded views of the internal components. The question asks the respondent to identify which are most similar based on the underlying structure of the internal system.

Question 7: Form and Function Representations of Technological Systems Engineering experts familiar with the nature of technological systems are able to identify correspondence between the form of a system and functional descriptions of that system such as electrical schematics or piping diagrams. The question addresses the transfer between a representation of the system form (how it looks) and a diagram representing the essential components and their interaction. A drawing of an automobile exhaust system is given and the 
respondent is asked to select which schematic block diagram best represents the system. Distractors include components out of order and incomplete or extra component interactions.

\section{Initial Results}

The initial pilot of the test was conducted in a general-education engineering course at Hope College. The course satisfies a general education laboratory course for non-STEM majors. A total of 42 students took the test. Of these students 48 percent were male and 52 percent female. All of these students are majoring in a discipline that is not a field of science, engineering, technology, or mathematics. The material on the test was not covered in the course at the time the test was given. The students had no opportunity to study or prepare, the test was given "cold."

Figure 1 shows the distribution of results for this group. The average of the class was $4.6 / 7$ or $66 \%$. The standard deviation was $1.5 / 7$. The median score was $5 / 7$ or $71 \%$. Of the 42 students $33 \%$, scored $5 / 7$ (715). A total of $60 \%$ of the group scored 70 or higher.

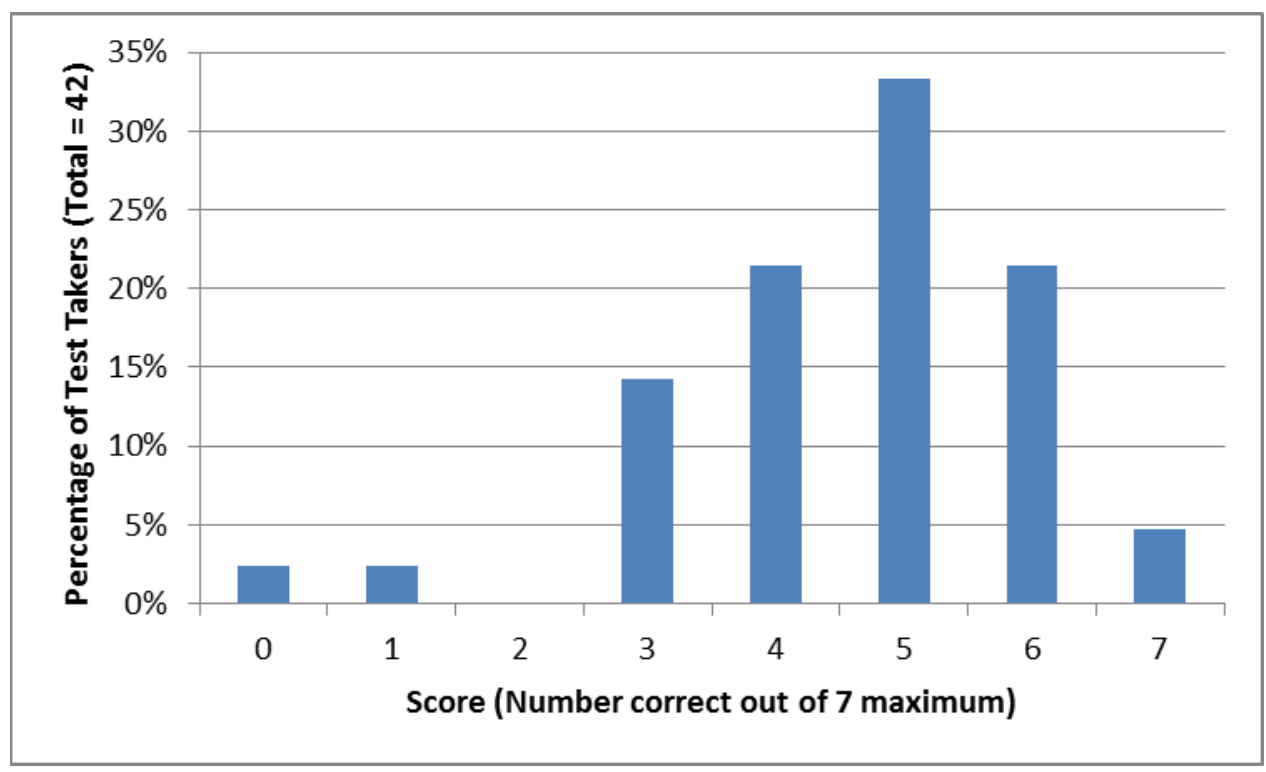

Figure 1: Distribution of Scores from Initial Use of Engineering Reasoning Test.

Interpreting the results in an absolute sense is not possible based on only this initial application of the test. Data do not yet exist for a comparison to what scores are obtained by engineering experts. However the general bell-curve shape of the distribution for this one group is encouraging for pursuit of additional test cases. The distribution shows that this initial test leads to a range of scores and can thus resolve different levels of engineering reasoning ability among the non-engineering group. The test did not result in a distribution skewed toward very low or very high scores. 
The results for each question are shown in Figure 2. It is apparent the question 4 proved the most challenging. In this question students had to select a component from a set of possible choices. The choice was to be informed by carrying out a simple calculation. The formula for the calculation was included in the question statement.

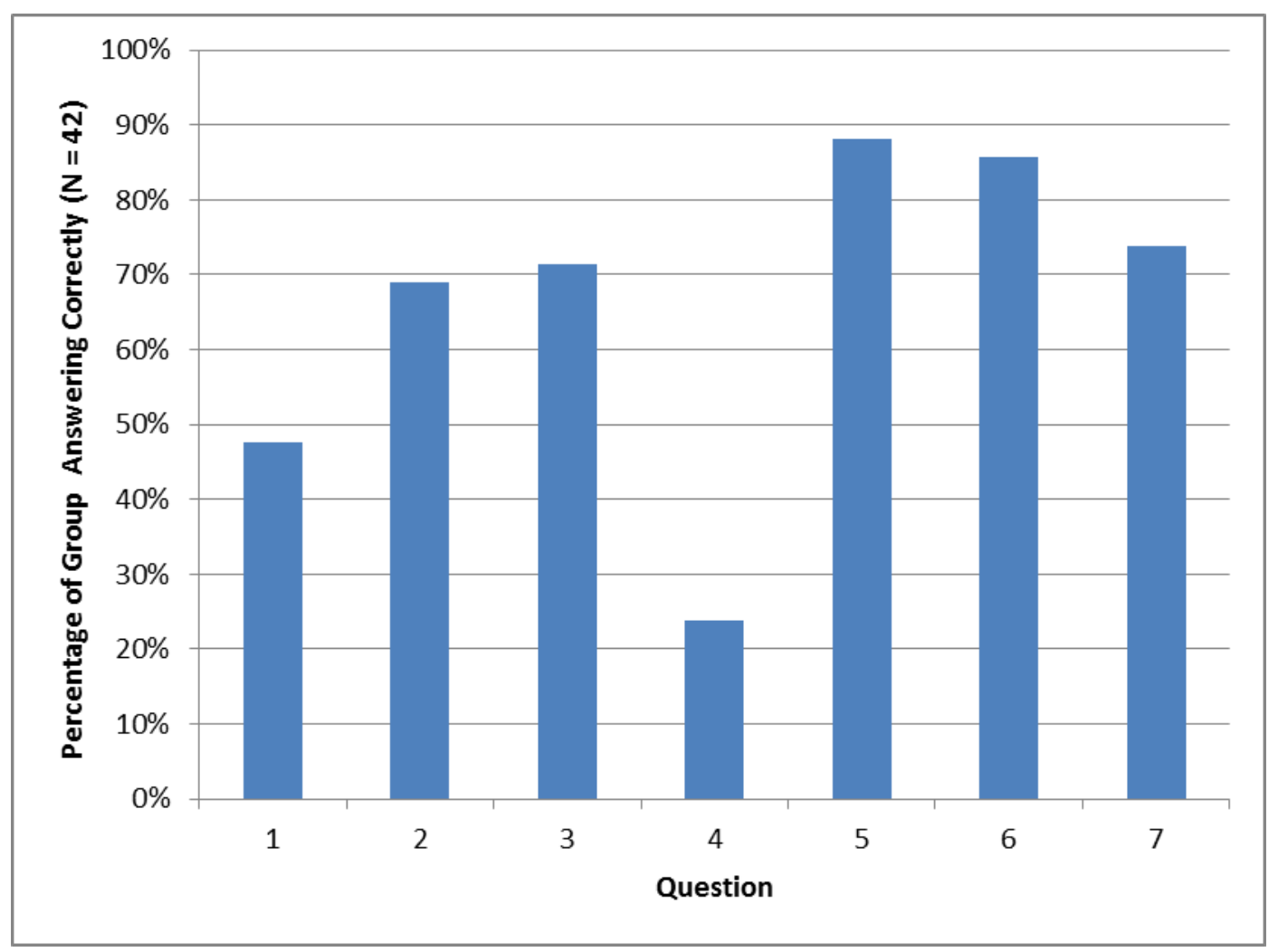

Figure 2: Percentage of Test Group Answering Correctly for Each Question.

\section{Conclusions and Future Work}

The goal of creating a general and broadly applicable assessment of engineering and technological literacy is a challenging task but one that might be addressed by focusing on the some of the thinking processes that are characteristic of engineering reasoning. This work has shown some encouraging preliminary results in developing an engineering reasoning test modeled after the ACT Science Reasoning Test. These tests focus on thinking skills rather than recall of facts by providing data and other background information within the body of the test question. Current work has shown that pilot questions based on this approach yield reasonable results when used by undergraduate non-engineering students. Future work will widen the number of student tested and will increase the number of questions to encompass a wide range of engineering reasoning skills. 


\section{Acknowledgement}

Some aspects of this work were supported by the National Science Foundation under award: DUE-1121464. Any opinions, findings, and conclusions or recommendations expressed in this material are those of the authors and do not necessarily reflect the views of the National Science Foundation.

\section{Bibliography}

1. Pearson G., and A. T. Young, Technically Speaking: Why All Americans Need to Know More about Technology. National Academies Press (2002).

2. Pearson G., and E. Garmire, Tech Tally: Approaches to Assessing Technological Literacy. National Academies Press, (2006).

3. David Hestenes, D., M. Wells, and G. Swackhamer, Force Concept Inventory, Physics Teacher, 30, 141 (1992).

4. Law School Admissions Test (LSAT), Law School Admissions Council, http://www.lsac.org/,(accessed December 1, 2015).

5. $\quad$ Miller Analogies Test (MAT) http://www.pearsonassessments.com/postsecondaryeducation/graduate_admissions/mat.html, (accessed December 1, 2015).

6. ACT Science Test, http://www.actstudent.org/testprep/descriptions/scidescript.html, (accessed December 1, 2015).

7. Krupczak, J.J, and M. Mina, “Work in Progress: An Approach to Engineering Literacy Emphasizing Components, Functions, and Systems,” Proceedings of the American Society for Engineering Education 2015 Annual Conference, Seattle, WA

\section{Appendix A: Example of Engineering Reasoning Test Question}

Due to copyright considerations the entire test is not included. Interested educators may obtain copies of the full test by contacting the authors. An example of one question is included below. This sample question begins on the next page. The answer is (c). 


\section{Engineering Reasoning Diagnostic Test}

\section{Hybrid Car Design}

Consider the design of a hybrid car. In this design a battery provides electrical energy to an electric motor which in turn drives the wheels. When the battery is depleted an internal combustion engine turns a generator which supplies electrical power to the motor and also recharges the battery.

Major Hybrid Car Components and Their Function

\begin{tabular}{l}
\hline Battery \\
(store electrical \\
energy)
\end{tabular}

Generator (convert energy of motion into electrical energy )

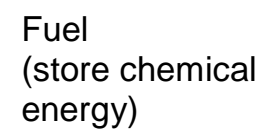

Electric Motor

(convert electrical energy into energy of motion )

1. Which of the diagrams on the next page shows the flow of energy in this system if the vehicle is in use (ie: being driven) after the battery has been depleted.
(a) Diagram 1
(b) Diagram 2
(c) Diagram 3
(d) Diagram 4
(e) None of the Above

Internal Combustion Engine (convert chemical energy of fuel into energy of motion ) 
(a) Diagram1

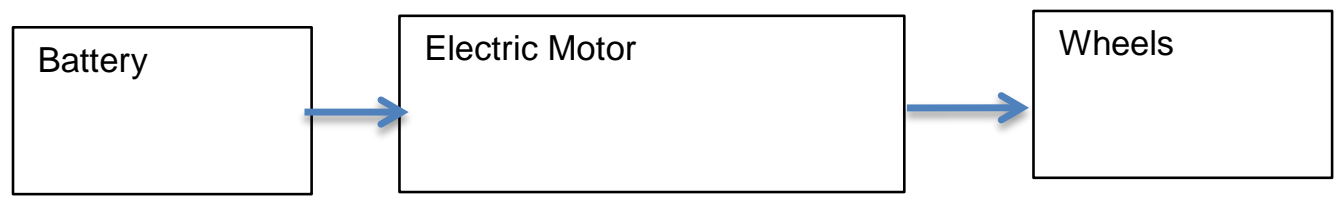

(b) Diagram 2

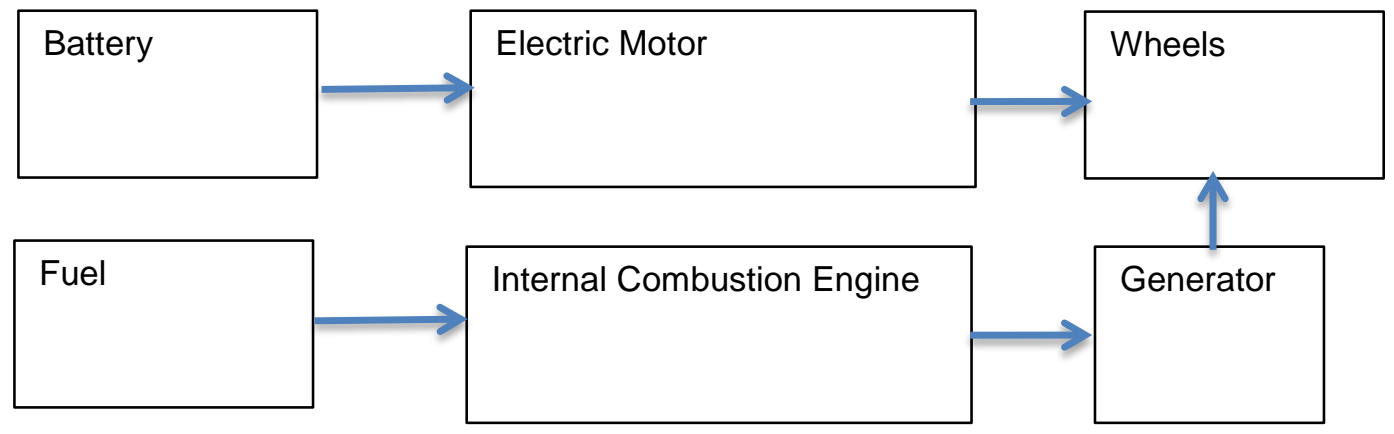

(c) Diagram 3

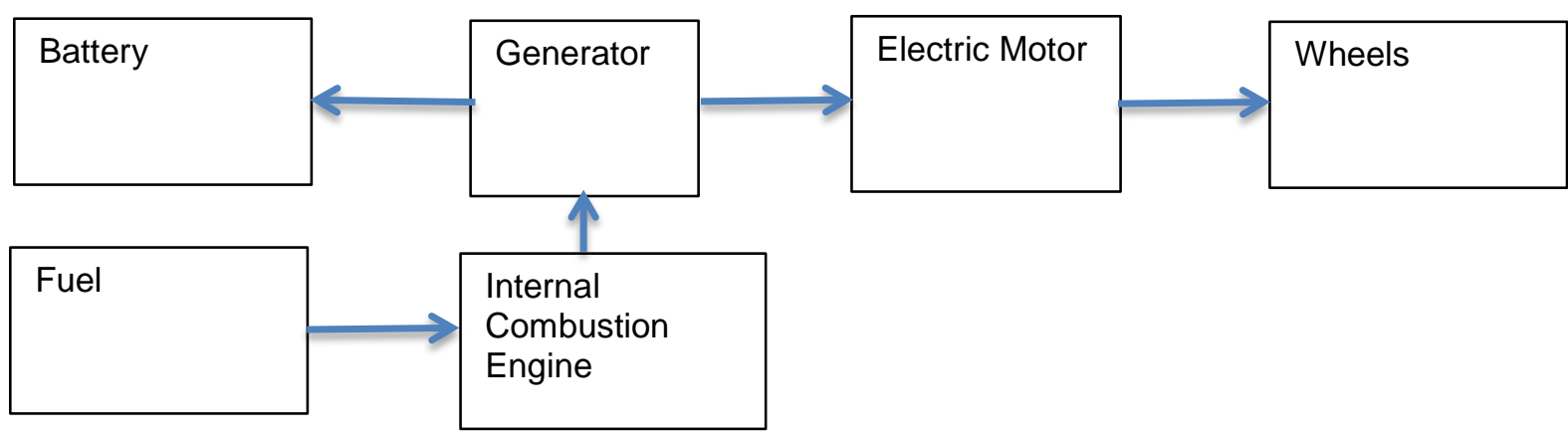

(d) Diagram 4

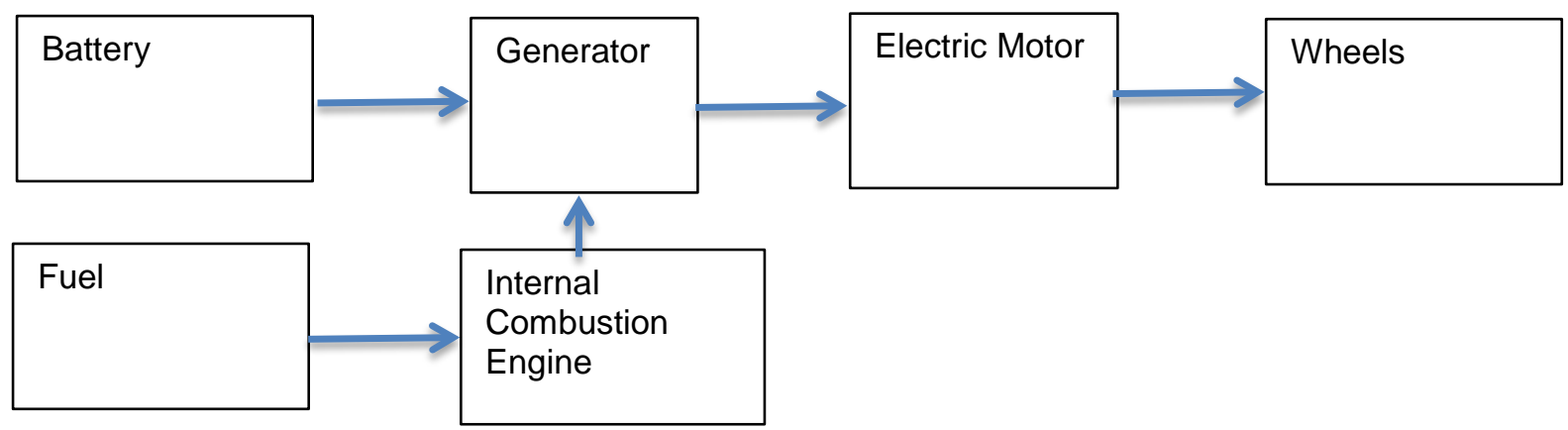

